\title{
ZOONOSES CAUSADAS POR PARASITAS INTESTINAIS DE CÃES E O PROBLEMA DO DIAGNÓSTICO
}

\section{S. Katagiri; T.C.G. Oliveira-Sequeira}

Universidade Estadual Paulista, Instituto de Biociências, Departamento de Parasitologia, CP 510, CEP 18618-000, Botucatu, SP, Brasil.

\section{RESUMO}

\begin{abstract}
Os últimos 20 anos foram marcados por um intenso progresso tecnológico e por importantes alterações culturais e sociais com reflexos tanto em saúde humana como animal. Entretanto, infelizmente, as zoonoses causadas por parasitas intestinais de cães não deixaram de ser um problema permanente de saúde pública nos países em desenvolvimento, e uma preocupação crescente nos países desenvolvidos. A eficácia e segurança dos novos produtos disponíveis para a prevenção e controle não impediram que enfermidades parasitárias emergissem como um sério problema em animais de companhia. Nesta revisão, foram consideradas as principais espécies de parasitas intestinais de cães, responsáveis por zoonoses, e os aspectos relativos ao diagnóstico coproparasitológico com o objetivo de contribuir para um maior comprometimento dos médicos veterinários no importante papel de agentes promotores de saúde pública e de difusores do conhecimento.
\end{abstract}

PALAVRAS-CHAVE: Cães, parasitas intestinais, diagnóstico, helmintos, protozoários.

\section{ABSTRACT}

ZOONOSESCAUSED BY DOG INTESTINAL PARASITES ANDTHE PROBLEMOF DIAGNOSIS. The last 20 years have witnessed a great technological progress along with important cultural and social changes that have improved some aspects of both human and animal health. However, zoonotic diseases caused by dog intestinal parasites persist as a commonplace public health concern in developing countries and as an increasing problem in developed ones. The effectiveness and security of the new drugs available for the prophylaxis and control of parasites has not hindered the emergence of parasitic infection as a serious problem in pets. This review aimed to examine some of the more important intestinal parasites of dogs involved in zoonotic diseases and to highlight issues associated with their diagnosis. The main purpose is to advise veterinarians in clinical practice concerning their importance as a public health agent in preventing and controlling dog zoonotic diseases through the correct use of antiparasite drugs and by educating pet owners.

KEY WORDS: Dogs, intestinal parasites, diagnosis, helminthes, protozoa.

Os cães foram a primeira espécie a ser domesticada e embora se desconheça o que motivou o homem pré-histórico nessa empreitada, atualmente, nenhuma outra espécie animal ocupa tantos e tão diversos papéis na sociedade humana, sendo inestimáveis os benefícios dessa convivência para a melhoria das condições fisiológicas, sociais e emocionais principalmente de crianças e idosos (Asano et al., 2004). Entretanto, por estarem envolvidos involuntariamente na transmissão de mais de 60 infecções zoonóticas (MACPHERson et al., 2005) e constituir uma importante fonte de infecção por parasitas, bactérias, fungos e vírus (Plant et al., 1996; Gefrray, 1999), a manifestação de todos os benefícios dessa convivência pode ser perdida se a saúde desses animais não for objeto de maiores cuidados.
A associação com o homem facilitou a dispersão desses animais por todos os continentes, assim, a população atual de cães é estimada em mais de 500 milhões de animais (MACPHERSON et al., 2005). Da mesma forma que os próprios hospedeiros, a maioria dos parasitas intestinais de cães têm distribuição cosmopolita. No entanto, o elenco de espécies e suas respectivas prevalências são afetados por fatores climáticos, hábitos culturais, recursos diagnósticos e nível de notificação (McCARTHY; MOORE, 2000).

Os parasitas intestinais estão entre os agentes patogênicos mais comumente encontrados em animais de companhia e constituem uma das principais causas de transtornos intestinais em cães (BLAGBURN et al., 1996). Os cães são parasitados por cerca de 17 espécies de trematódeos, 17 de cestódeos, 20 de 
nematódeos e 1 acantocéfalo (Eguía-Aguilar et al., 2005) e por um grande número de protozoários. No entanto, o volume de informações disponíveis sobre cada espécieémais ou menos abundante dependendo da importância dessa espécie para os próprios cães ou do seu envolvimento na produção de infecções humanas ou de espécies domésticas exploradas comercialmente. Dessa forma, Ancylostoma spp., Toxocara canis, Giardia sp. e Cryptosporidium spp., que infectam o homem (GENNARI et al., 1999) e Neospora caninum (MOore, 2005), por ser uma das principais causas de aborto bovino em todo o mundo, têm recebido maior atenção.

As infecções parasitárias acometem cães de todas as idades, mas usualmente são mais prevalentes em filhotes; isso se deve principalmente ao fato de que muitos parasitas utilizam vias de transmissão que expõem especificamenterecém-nascidos ou neonatos e também porque os cães jovens não respondem imunologicamente de forma eficaz (RAMÍREZ-BARRIOS et al., 2004).

Ancylostoma caninum é um nematódeo hematófago do intestino delgado de cães cuja principal forma de infecção é efetuada pela passagem de larvas pelo leite de cadelas lactantes. Os animais jovens são os mais freqüentemente acometidos, porém ao longo da vida do animal a infecção continua a ocorrer pela penetração cutânea e pela ingestão de larvas juntamente com alimentos e água (BowmAN et al., 2002). Além disso, larvas encistadas na musculatura podem ser reativadas em situações de queda de imunidade ou de administração de anti-helmínticos, promovendo a recolonização periódica do intestino (KALKOFEN, 1987).

Nos cães, a gravidade das infecções provocadas por estes nematódeos depende da via de infecção, do número de formas infectantes e da resposta imune desses hospedeiros. Em cães jovens, a passagem de larvas pelo leite pode ter conseqüências fatais ou ser responsável pela produção de quadros de anemia hemorrágica aguda ou crônica acompanhada de diarréia que pode conter sangue e muco. Em cães adultos podem causar deficiência de ferro e anemia hipocrômica microcítica (URQUHART et al., 1996).

Uma segunda espécie do gênero que pode infectar os cães é Ancylostoma braziliensis. Trata-se de espécie menos prevalente, cujos efeitos relacionados à hematofagia causam transtornos semelhantes a $A$. caninum. No entanto, por ser menos voraz é também menos patogênica.

Larvas infectantes de ambas as espécies de Ancylostoma podem penetrar na pele de seres humanos causando a dermatite serpiginosa ou Larva Migrans Cutânea (LMC), conhecida popularmente por "bichogeográfico". A penetração dessaslarvas na pele provoca, inicialmente, uma reação intensamente pruriginosa e constitui um importante problema de saúde-pública. No Brasil, não se dispõem de dados sobre a prevalência de LMC e os poucos dados disponíveis se restringem ao relato de casos com apresentação clínica excepcional (VelHo et al., 2003), ou de surtos em grupos populacionais específicos como escolas e creches (ARAúJo et al., 2000). Não obstante, em diferentes localidades foram registradas elevadas taxas de contaminação ambiental por ovos e larvas de Ancylostoma em locais públicos e de recreação infantil (GUIMARÃEs et al., 2005; SANTARÉM et al., 1998; Araújo et al., 1999; COSTA-CruZ et al., 1994).

Na Austrália a infecção humana por adultos de $A$. caninum foi identificada recentemente como causa de uma "enterite eosinofílica", caracterizada por dor e distensão abdominal, diarréia, perda de peso e hemorragia retal (ROBERTSON; THOMPSON, 2002). A via de infecção que leva ao estabelecimento de vermes adultos no intestino humano ainda não foi demonstrada com precisão. Entretanto, nem a exposição percutânea ou as inoculações, experimentais ou acidentais, por essa via foram capazes de reconstituir os sintomas abdominais, a acentuada eosinofilia ou a produção de anticorpos. Por conseguinte, LANDMANN; PROCIV (2003) sugeriram que a presença do verme adulto no lume do intestino humano seria uma conseqüência da ingestão de larvas.

A ubiqüidade de $A$. caninum e a dificuldade de diagnosticar essa patologia fazem crer que a ausência dessa síndrome em outros países seja devida à falta de diagnóstico (McCarthy; MoOre, 2000).

Assim como Ancylostoma spp., Toxocara canis é freqüentemente encontrado no intestino delgado de cães. A principal via de infecção é pela passagem transplacentária de larvas que se encontram encistadas nos tecidos das cadelas prenhes. Por isso, e também por causa da ubiqüidade desse verme, aproximadamente $80 \%$ dos cães com menos de seis semanas de idade possuem exemplares de Toxocara em seus intestinos, podendo ou não eliminar os ovos nas fezes (ROBERTSON; THOMPSON, 2002) e morrer em conseqüência do parasitismo. Embora seja uma infecção freqüente em animais jovens, cães adultos podem permanecer susceptíveis e contribuir para a contaminação ambiental (OvergaAuw, 1997). Nos cães, os efeitos da infecção por T. canis dependem da idade do animal, do número, localização e estágio de desenvolvimento dos vermes (PARSONS, 1987). Em infecções maciças, a migração de grande número de larvas pelos pulmões pode provocar tosse, aumento da freqüência respiratória e corrimento nasal espumoso; os vermes adultos no intestino podem causar obstrução intestinal, dos ductos colédoco e pancreático (URQUHART et al., 1996).

A infecção humana por T. canis ocorre pela ingestão de ovos larvados presentes no solo poluído, em fômites e em mãos contaminadas com fezes de animais parasitados. O parasita não atinge a maturi- 
dade nesses hospedeiros, mas as larvas permanecem vivas migrando erraticamente nos órgãos internos, produzindo uma patologia denominada Larva Migrans Visceral (LMV) (PARSONS, 1987), ou invadindo o globo ocular e causando a Larva Migrans Ocular (LMO). A infecção humana por T. canis é a mais comum zoonose parasitária transmitida por cães nos Estados Unidos, onde anualmente a infecção é causa de centenas de casos de cegueira unilateral e de outras formas inespecíficas de enfermidades em crianças (ROBERTSON et al., 2000). Crianças entre 2 e 5 anos de idade são mais freqüentemente infectadas. A convivência direta com cães não é indispensável para que ocorra a infecção, basta que se entre em contato com o solo contaminado com excremento de cães parasitados (REY, 1992).

Entres aspectos interessantes em relação à LMV destacam-se a freqüência relativamente baixa decães infectados com T. canis registrada nos diferentes estudos realizados no Brasil - 11,70 (CÔRTES et al., 1988), 8,49 (Gennari et al., 1999), 5,54 (Oliveira-SEQueira et al., 2002) - e as elevadas soroprevalências detectadas em crianças (MoreIRA-SILVA et al., 1998; CAMPOS-JúNIOR et al., 2003). Vários aspectos relacionados à biologia e epidemiologia desse parasita concorrem para produzir essa situação: a grande fecundidade das fêmeas; a resistência dos ovos que permanecem viáveis no ambiente por vários meses (JoRDAN et al., 1993) e o fato do parasitismo ser mais freqüente em animais jovens, porque os animais muito jovens são uma categoria freqüentemente minoritária nos levantamentos epidemiológicos (FonTANARROSA et al., 2005).

Dos nematódeos parasitas de cães, Ancylostoma spp. e T. canis certamente são os mais ubíquos e prevalentes. A grande tolerância dos estágios de vida livre às diferentes condições ambientais é a mais provável explicação para a ampla distribuição geográ-fica desses parasitas. Por outro lado, as prevalências freqüentemente elevadas desses parasitas parecem estar relacionadas a diferentes estratégias.

No caso de Ancylostoma, como os animais permanecem susceptíveis às infecções ao longo de toda a vida (KALKOFEN, 1987), tanto as larvas presentes no ambiente como as retidas nos tecidos podem alcançar periodicamente o intestino recolonizando-o com vermes adultos. Com isso, teoricamente, toda a população de cães contribui de forma contínua para a contaminação ambiental.

No caso de T. canis, embora os animais adultos possam albergar vermes adultos no intestino e eliminar ovos nas fezes, a contaminação ambiental parece ser, principalmente, devida a grande fecundidade das fêmeas (HABLUETZEL et al., 2003) e à elevada resistência dos ovos no ambiente (OvERGAAUw, 1997).

No Brasil, os dados de prevalência dessas duas parasitoses em cães são escassos e se restringem a poucos locais, geralmente, sede de instituições de pesquisa. Na Cidade de São Paulo, no período de 1980 a 1985 (CÔRTEs et al., 1988), as prevalências de $59,83 \%$ e 11,70\% para ancilostomídeos e toxocarídeos respectivamente, foram mais elevadas que as registradas no período de 1991 a 1995 por GenNARI et al. (1999); neste último período, as prevalências obtidas foram de 20,40\% para Ancylostoma spp. e 8,49\% para T. canis. Estes dados, à semelhança do que vem sendo observado em países desenvolvidos sugerem uma redução temporal nas prevalências desses vermes. No entanto essa informação não pode ser extrapolada para outras regiões do país, mesmo para as que se encontram no mesmo nível de desenvolvimento. Em Balneário Cassino, RS, SCAINI et al. (2003) relataram que $86,1 \%$ das amostras de fezes de cães coletadas em vias públicas estavam contaminadas por ovos e larvas de helmintos, das quais $71,3 \%$ por Ancylostoma spp. e $3 \%$ por $T$. canis.

Os principais cestódeos parasitas gastrintestinais de cães pertencem às famílias Dilepididae (Dipylidium caninum) e Taeniidae (Taenia sp. e Echinococcus sp.), e a maioria deles é bastante adaptada a esses hospedeiros não lhes causando grandes transtornos (GEORGI, 1987).

Na família Dilepididae, Dipylidium caninum, espécie mais comumente encontrada no intestino delgado de cães, tem importância para a saúde dos cães e constitui uma zoonose (Molina et al., 2003). Como o potencial de transmissão entre os cães é função da densidade populacional de pulgas, que são hospedeiras intermediárias do parasita, as prevalências são variáveis, podendo ser tão elevadas quanto $60 \%$, conforme registrado em algumas localidades (Eguía-Aguilar et al., 2005).

O parasitismo por D. caninum é considerado pouco patogênico; os cães podem tolerar centenas de vermes adultos sem manifestarem sintomatologia. A infecção humana resulta da ingestão acidental de pulgas parasitadas. Em crianças o parasita pode causar desconforto ou dores abdominais, diarréia, prurido anal e irritabilidade nervosa (REY, 1992).

Um aspecto que se destaca quando se examina a literatura relativa à prevalência de $D$. caninum é a discrepância das prevalências registradas: extremamente elevadas quando o diagnóstico é feito pelo encontro dos vermes à necropsia (EGUíA-AgUILARetal., 2005) e muito baixas quando detectada por exames coproparasitológicos (GENNARI et al., 1999; OLIVEIRA-SEQueira et al., 2002; AsAno et al., 2004; RAmíreZ-BARRIos et al., 2004). A constatação de discrepâncias na prevalência desse parasita, em uma mesma região geográfica, devida à metodologia diagnóstica, tem incentivado a pesquisa de métodos sorológicos para diagnóstico dessa parasitose (SHIN; LiAO, 2002).

A maioria das espécies do gênero Echinococcus tem por hospedeiro definitivo os canídeos domés- 
ticos ou silvestres e as larvas, ou cistos hidáticos, se desenvolvem em diversas espécies de mamíferos, inclusive no homem. Os vermes adultos não são patogênicos para os cães, já que estes hospedeiros podem suportar um elevado número de parasitas sem apresentar sintomatologia clínica (RAETHER; HÄNEL, 2003). Por outro lado, as formas larvais desses parasitas infectam animais domésticos ou o homem, fazendo com que o parasitismo constitua um grave problema tanto econômico como de saúde pública.

À exceção do agente etiológico da hidatidose unilocular, Echinococcus granulosus, que se distribui de forma descontínua em todos os continentes, as demais espécies têm uma distribuição mais restrita (ECKert; Deplazes, 2004). Das espécies que têm o cão por hospedeiro definitivo, apenas E. granulosus e E. vogeli ocorrem no Brasil. Os casos de infecção humana por E. vogeli são registrados exclusivamente nos Estados da Região Norte (MoraEs et al., 2003).

Com relação a E. granulosus, a infecção é comum no Estado do Rio Grande do Sul, onde o cão doméstico é o principal hospedeiro definitivo e os bovinos e ovinos os hospedeiros intermediários (FARIAS et al., 2004). Os dados sobre as prevalências da infecção canina são escassos. Recentemente a detecção de cães parasitados $(7,7 \%)$ ou com sorologia positiva para esse cestódeo $(13,8 \%)$ em zona urbana no Rio Grande do Sul (HoFfMMAN, 2001) chamou a atenção para a possibilidade de urbanização da infecção humana. Embora no Brasil a hidatidose ainda seja considerada uma enfermidade tipicamente rural essa preocupação se justifica pelo fato de que na Austrália a hidatidose, antes uma enfermidade rural, converteu-se em um problema de saúde pública emergente em função do estabelecimento de focos urbanos da infecção (ROBERTSON; THOMPSON, 2002).

De acordo com GEORGI; GEORGI (1992) os cães são parasitados por 8 espécies de Taenia, das quais 5 têm uma ampla distribuição geográfica. No Brasil, foram registradas as espécies Taenia hydatigena, Taenia taeniformis e Taenia multiceps (COSTA et al., 1986).

As espécies de Taenia que têm o cão como hospedeiro definitivo são pouco patogênicas para este hospedeiro, entretanto as formas larvais de algumas espécies podem se desenvolver em animais domésticos ou mesmo apresentar potencial zoonótico (ING et al., 1998). Os cenuros de Taenia multiceps se desenvolvem predominantemente em ruminantes e as infecções humanas parecem grandementeconfinadas ao continente africano, com poucos casos registrados na França, Inglaterra e Américas do Norte e do Sul (RAETHER; HëNEL, 2003).

Uma das dificuldades de se investigar, especificamente, as prevalências de infecções por taenídeos em cães, é a impossibilidade de se distinguir morfologicamente os ovos das diferentes espécies de Taenia e de Echinococcus (CABRERA et al., 2002).
Os protozoários parasitas gastrintestinais decães incluem flagelados, como Giardia, e coccídios, com os gêneros Isospora, Hammondia, Cryptosporidium, Sarcocystis e Neospora (GeOrGI; GeORGI, 1992).

São reconhecidas cinco espécies de Giardia que habitam o trato intestinal de virtualmente todas as classes de vertebrados, porém Giardia duodenalis (= Giardia intestinalis $=$ Giardia lamblia $)$ é a única espécie encontrada em seres humanos e na maioria dos mamíferos domésticos e silvestres (THOMPson et al., 2002).

G. duodenalis tem distribuição global e de acordo com a Organização Mundial de Saúde há cerca de 200 milhões de pessoas infectadas com giardíase sintomática e 500 mil novos casos registrados anualmente na Ásia, África e América Latina (WHO, 1996).

Em países desenvolvidos, G. duodenalis tem sido reportado como o parasita entérico mais comum de cães domésticos (Buggetal., 1999). Nos levantamentos realizados em países em desenvolvimento, quando nãoéo mais prevalente, figura entre os 3 mais comuns (Gennari et al., 1999; Oliveira-SequeIra et al., 2002; Ponce-Macotela et al., 2005). Ainda assim, considera-se que a prevalência de Giardia em animais de companhia éfreqüentemente subestimada por causa da baixa sensibilidade dos métodos convencionais de diagnóstico, em virtude dos parasitas ocorrerem em níveis subclínicos e da eliminação de cistos ser naturalmente intermitente (McGLADE et al., 2003).

Atérecentemente, a transmissibilidade zoonótica de Giardia entre cães e seres humanos era considerada uma possibilidade carente de confirmação. Após a introdução das técnicas moleculares de investigação constatou-se que G. duodenalis é uma espécie que inclui um complexo de 8 genótipos principais, denominados "assemblages" (Monis et al., 2003). Todos os isolados humanos investigados foram identificados como pertencentes aos "assemblages" A e B e os genótipos exclusivos de cães foram identificados como assemblages C e D. No entanto, os estudos de epidemiologia molecular têm revelado que os cães podem ser infectados tanto com os seus próprios genótipos de Giardia (assemblages C e D), bem como com genótipos zoonóticos (assemblage AI e BIII) (THOMPson, 2004).

No que se refere aos próprios cães, o significado patogênico das infecções por Giardia é mínimo e, embora a transmissão zoonótica deste protozoário seja inquestionável, as implicações do parasitismo dos cães em termos de saúde pública não estão estabelecidas (THOMPSON, 2004).

Embora os cistos provenientes de fezes humanas sejam os principais responsáveis pelos casos de infeç̧ão humana, dados obtidos em diferentes regiões sugerem a necessidade de se examinar a transmissibilidade deste protozoário entre hospedeiros que compartilham a mesma área geográfica em regiões endêmicas. Na Índia, onde Giardia ocorre tanto em 
humanos como em cães, 20\% dos cães parasitados foram encontrados portando o genótipo zoonótico, principalmente o "assemblage" A (THOMPSON, 2004). No Brasil, ogrande número deindivíduos que vivem em comunidades onde a precariedade de habitação favorece a convivência promíscua, principalmente entre crianças e cães; a falta de saneamento básico e as elevadas prevalências de Giardia nesses hospedeiros fazem com que a transmissão zoonótica desse protozoário seja uma possibilidade que não pode ser negligenciada.

Quanto aos coccídios intestinais de cães, a ocorrência de um hospedeiro intermediário é obrigatória no ciclo evolutivo das espécies de Sarcocystis, Neospora e Hammondia, facultativa no caso de Isospora eausente no ciclo de Cryptosporidium (Bowman et al., 2002). As características morfológicas de alguns oocistos de coccídios que têm o cão como hospedeiro definitivo, não são suficientes sequer para a identificação genérica desses parasitas, como é o caso de Neospora e Hammondia.

Oscães são hospedeiros definitivos de um grande número de espécies de Sarcocystis cujos hospedeiros intermediários incluem mamíferos domésticos e silvestres e também aves. As espécies de Sarcocystis que têm o cão por hospedeiro definitivo são consideradas apatogênicas para este hospedeiro (DUBEY et al., 2000).

Cryptosporidium spp. é um coccídio intestinal que afeta diversas espécies de animais domésticos e também o homem. São consideradas válidas 13 espécies de Cryptosporidium parasitas de mamíferos, com mais de 30 genótipos distintos já descritos (XIAO; RYAN, 2004). De acordo com os estudos moleculares mais recentes, dois genótipos são mais comumente encontrados em humanos: o genótipo humano (ou tipo I; reconhecido como Cryptosporidium hominis) eo genótipo bovino (ou tipo II; Cryptosporidium parvum). Ao que tudo indica, o genótipo humano é restrito ao homem, mas o genótipo bovino, além de infectar o homem, é infectante para outras espécies de mamíferos (Monis; Thompson, 2003). Conseqüentemente, os cães infectados podem constituir uma fonte de infecção para o homem, especialmente, para indivíduos imunocomprometidos (MILSTEN; GolDSMID, 1995). Além disso, outras espécies de Cryptosporidium, incluindo C. canis, têm sido encontradas em amostras clínicas provenientes tanto de indivíduos imunocompetentes como de imunocomprometidos. De acordo com MonIS; THOMPSON (2003) a freqüência dessas infecções é mais baixa e envolve indivíduos com enfermidades concomitantes ou que vivem em condições que aumentam a susceptibilidade a essas infecções.

Em cães, as infecções por Cryptosporidium são mais severas em animais jovens e podem ser exacerbadas por estresse e imunossupressão (ROBERTSON et al., 2000). A criptosporidiose foi diagnosticada pela primeira vez em cães em 1983 e tanto no cão como em outros hospedeiros o parasitismo provoca atrofia das vilosidades intestinais (WILSON, 1983).

Muitas infecções de cães com C. parvum são assintomáticas, porém infecções simultâneas com o vírus da cinomose podem levar à doença clínica. Apesar de poucos cães eliminarem oocistos, as elevadas taxas de soropositividade encontradas sugerem um histórico de exposição prévia (ROBERTSON et al., 2000).

Embora a maioria dos parasitas gastrintestinais decães apresente distribuição cosmopolita, de modo geral, são mais prevalentes em países tropicais e subtropicais (CORDERO Del CAMPILlo; Rojo, 1999) e naqueles em que as condições sócio-econômicas da população são precárias (Gomeset al., 2004). Todavia, mesmo em países desenvolvidos as zoonoses parasitárias caninas têm sido motivo de preocupação. As imunodeficiências de modo geral, e em especial a aids; a inserção cada vez mais diversificada e ampla dos cães na sociedade e o desenvolvimento de tecnologias que melhoraram as condições de diagnóstico e investigação puseram em evidência, especialmente nos últimos 20 anos, algumas parasitoses, freqüentemente referidas como "emergentes" ou "reemergentes" (Ambroise-Thomas, 2000). Ao analisar essa conjuntura THOMPSON (1999) ressaltou que compete aos médicos veterinários deste novo milênio o diagnóstico, a estruturação de um sistema de investigação e acompanhamento epidemiológico e a transferência de conhecimento através da educação sanitária para que essas parasitoses possam ser adequadamente controladas.

O grande desenvolvimento da quimioterapia antiparasitária a partir do descobrimento dos benzimidazóis (CHAN, 1997), tem sido destacado como uma das razões para se dispensar o exame coproparasitológico em clínica de pequenos animais. Além disso, é fácil constatar que a coproscopia é a atividade de menor prestígio tanto em laboratório clínico humano como veterinário, pois lida com material mal-cheiroso, de aspecto nauseabundo e para a qual houve muito pouco desenvolvimento tecnológico. No entanto, apesar da eficácia e segurança dos produtos disponíveis para prevenção e controle das enteroparasitoses, em todo o mundo existem evidências de que as infecções por parasitas emergentes ou reemergentes estão aumentando nos animais de companhia (FAYER, 2000).

Atualmente, apesar da disponibilidade de técnicas imunológicas e moleculares para o diagnóstico de algumas parasitoses intestinais humanas, dois aspectos restritivos à utilização dessas técnicas devem ser observados. Em primeiro lugar, que a disponibilidade de muitas dessas técnicas ainda é limitada aos laboratórios de pesquisa; em segundo, que em muitos casos não se justifica a substituição 
das técnicas coproparasitológicas convencionais, que em muitas situações continuam sendo as de eleição pela simplicidade, sensibilidade ebaixo custo (THOMPSON, 1999). Apesar da identificação dealgumas das parasitoses emergentes só ter sido possível graças ao emprego de métodos moleculares, a abolição dos eficazes métodos convencionais não se justifica (THOMPSON, 1999). O exame microscópico de fezes ainda é a base do diagnóstico de grande número de enfermidades parasitárias, pois possibilita a detecção das formas evolutivas de helmintos e protozoários com $100 \%$ de especificidade.

Como existem diferentes métodos para o processamento de amostras fecais para o exame microscópico, é necessário observar algumas características tais como sensibilidade, facilidade de execução e custo (FOREYT, 1989). Além da sensibilidade intrínseca do método, é necessário considerar os fatores extrínsecos que podem influenciar na eficiência de um determinado método diagnóstico. $\mathrm{O}$ volume da amostra examinada, o tempo de coleta, o uso de líquidos conservantes e as condições de envio ao laboratório também podem influenciar os resultados dos exames; amostras velhas ou precariamente preservadas são causas comuns de erros diagnósticos (AsH; ORIHEL, 1987).

As características físicas das fezes são o primeiro aspecto a ser observado, pois podem sugerir a presença de organismos patogênicos. No que diz respeito aos parasitas, pode-se dizer, de modo geral, que em fezes diarréicas encontram-se predominantemente trofozoítas de protozoários, enquanto que em fezes formadas, predominam cistos; ovos e larvas de helmintos podem ser encontrados em fezes de qualquer consistência (AsH; ORIHEL, 1987). No que se refere aos parasitas intestinais de cães, o exame macroscópico das fezes pode revelar a presença de nematódeos adultos e, principalmente, proglotes de cestódeos. A importância desse exame pode ser ilustrada pela constatação de que em Taiwan a prevalência da dipilidiose em cães errantes, avaliada pelo exame microscópico de fezes, foi de $0.35 \%$ e chegou a $63,3 \%$ quando avaliada pelo exame macroscópico das fezes (SHIN; LIAO, 2002).

As amostras de fezes a serem processadas pelos diferentes métodos coproparasitológicos podem ser utilizadas ainda frescas ou preservadas em substâncias fixadoras. As diferentes classes de substâncias fixadoras disponíveis têm a função de preservar as características morfológicas dos parasitas aumentando a chance de que eles sejam detectados e corretamente identificados (GARCIA, 1995).

O exame microscópico de fezes consiste em reconhecer as formas evolutivas típicas dos parasitas, distinguindo-as dos demais constituintes do material fecal. Dessa forma, independentemente da técnica coproparasitológica empregada, o exame microscópico apresenta valor preditivo positivo igual a unidade (=1), ou seja não detecta falso-positivos. Por outro lado, a sensibilidade analítica do exame depende da técnica a que as amostras foram submetidas para se obter as preparações microscópicas. Os métodos mais comumente empregados no processamento de amostras fecais incluem a diluição de uma pequena quantidade de fezes para o exame direto e os recursos de flutuação e sedimentação para concentração dos elementos parasitários (GARCIA, 2001). Considerando a diversidade de parasitas que eliminam formas evolutivas nas fezes, é fácil entender a razão pela qual nenhum desses métodos, quando utilizado isoladamente, é suficientemente sensível para detectar todos os possíveis parasitas que infectam um determinado hospedeiro. Assim sendo, é importante conhecer as utilidades e limitações dos métodos disponíveis.

O exame direto de fezes a fresco é realizado primariamente para detectar formas móveis de parasitas, tais como os trofozoítas de protozoários e larvas de helmintos. De acordo com Estevez; Levine (1985), trata-se de um método simples e rápido, cujos resultados positivos são tão válidos quanto os obtidos com métodos de concentração. O exame direto tem como principais limitações o fato de avaliar uma quantidade muito pequena de fezes e a dificuldade de se examinar amostras acondicionadas com conservantes, pois os parasitas morrem e com isso perdem a motilidade (Bowman et al., 2003). Embora possibilite a detecção de ovos e/ou larvas de helmintos, cistos ou oocistos de protozoários, estes são mais comumente encontrados quando se utilizam técnicas de concentração. Por tudo isso, as técnicas coproparasitológicas que empregam procedimentos de concentração acabaram substituindo o exame direto de fezes a fresco na rotina diagnóstica.

Os métodos de concentração por flutuação (gravitacional ou por centrifugação) se baseiam na diferença de densidade específica (DE) entre as formas evolutivas dos parasitas, os detritos fecais e a solução empregada para flutuação (DRYDEN et al., 2005). De acordo com esses autores, as soluções mais usadas para flutuação incluem as soluções de: cloreto de sódio $(\mathrm{DE}=1,18)$; açúcar (solução de Sheather, $\mathrm{DE}=1,27)$; nitrato de sódio ( $\mathrm{DE}=1,18)$; sulfato de magnésio $(\mathrm{DE}=1,20)$ e sulfato de zinco $(\mathrm{DE}=1,20)$.

Como a densidade específica da maioria das formas evolutivas dos parasitas situa-se entre 1,05 e 1,23 (DAVID; LiNDQUIST, 1982), o emprego de soluções com alta densidade específica permite a separação de cistos de protozoários, oocistos de coccídios e de ovos e larvas de helmintos, fazendo com que possam ser recuperados na superfície enquanto os detritos permanecem no fundo do tubo. Com isso, a preparação examinada em microscópioé mais limpa que a obtida pela concentração por sedimentação. As preparações obtidas com os métodos de flutu- 
ação devem ser examinadas prontamente porque as soluções empregadas produzem distorções nas formas parasitárias dificultando a sua identificação. De acordo com DRYDEN et al. (2005) os métodos mais empregados para a detecção de ovos e cistos de parasitas em fezes de pequenos animais são os que utilizam a flutuação como método de concentração.

Além de exigir o pronto exame das preparações, outra limitação dos métodos de concentração por flutuação é a baixa sensibilidade em detectar formas evolutivas de parasitas que apresentam DE mais elevada que a das soluções saturadas empregadas. Ovos de Physaloptera sp. e de Taenia spp., cujas densidades específicas são de 1,23761 e de 1,2251 (DRYDEN et al., 2005), podem não flutuar nessas preparações dando resultados falso-negativos. Nestes casos, as técnicas de sedimentação podem ser mais indicadas.

As técnicas que utilizam a sedimentação para concentração de formas evolutivas de parasitas são particularmente recomendadas para detectar infecções por helmintos que eliminam ovos pesados (operculados ou muito densos), mas também permitem a recuperação de cistos e oocistos de protozoários e de larvas de helmintos (GARCIA, 2001).

A sedimentação pode ser obtida de forma natural (gravitacional), por centrifugação ou por meios químicos (Truant et al., 1981).

A sedimentação por força gravitacional tem sido particularmente utilizada para detectar ovos operculados e de trematódeos como Schistosoma spp. (RABELLo et al., 1992). Dentre as vantagens do método destacam-se a simplicidade e o fato de não alterar a viabilidade dos ovos. As limitações incluem a produção de uma preparação contendo grande quantidade de detritos (que dificulta a identificação dos parasitas) e o longo tempo requerido para a sedimentação espontânea. A sedimentação por centrifugação abole apenas este último inconveniente.

A filtração prévia das amostras e a adição de formol-éter ou de acetato de etila às amostras de fezes facilitam o exame microscópio do sedimento, pois produzem uma preparação mais limpa. De acordo com TRUANT et al. (1981) a adição de formol-éter não provoca distorção nos organismos permitindo, inclusive, a identificação de formas evolutivas de helmintos e protozoários. O acetato de etila tem sido utilizado em substituição ao formol-éter com a vantagem de ser menos inflamável e perigoso.

O diagnóstico coproparasitológico tem sido uma das áreas da patologia clínica que tem evoluído mais lentamente, sendo uma das mais carentes de instrumentação, automação e desenvolvimento tecnológico (PERry et al., 1990). Entretanto, de acordo com estes autores, a partir de 1978, surgiram nos Estados Unidos vários dispositivos para concentração de amostras fecais e kits diagnósticos tornaram-se comercialmente disponíveis auxiliando na padroni- zação dos métodos de concentração, dando maior consistência à metodologia diagnóstica e com isso melhorando a deteç̧ão de parasitas.

Outra vantagem é que muitos desses kits são sistemas fechados nos quais as amostras são processadas com um mínimo de manipulação, tornando a atividade diagnóstica mais limpa, biologicamente segura e praticamentelivre dos odores desagradáveis característicos (GARCIA, 2001). Esses sistemas foram desenvolvidos originalmente para o diagnóstico de parasitoses intestinais humanas e embora introduzam diferentes procedimentos para a concentração dos parasitas, a maioria emprega o método original de sedimentação com algumas modificações (PERRY et al., 1990).

No Brasil, o conjunto comercial há mais tempo disponível é o Coprotest ${ }^{\circledR}$ (NL Comércio Exterior Ltda) (Cerqueira, 1988; Amato-Neto et al., 1989), desenvolvido para o diagnóstico coproparasitológico humano utilizando uma única amostra de fezes. Atualmenteé disponível sobuma nova denominação de Paratest ${ }^{\grave{O}}$ (Diagnostec - Comércio de Produtos Científicos), com modificações que possibilitam a colheita de três amostras seriadas em um único frasco coletor.

Recentemente, um outro kit comercial denominado TF-Test ${ }^{\mathrm{O}}$ (Immunoassay Comércio e Indústria Ltda), delineado para coleta seriada de amostras em três tubos, tornou-se disponível no mercado nacional. As amostras são tratadas com acetato de etila e detergente e processadas por dupla filtragem e centrifugação antes de serem examinadas ao microscópio óptico (Gomes et al., 2004).

A maioria dos estudos sobre a eficiência comparativa das técnicas coproparasitológicas convencionais e dos kits comerciais demonstram haver uma certa equivalência entre os métodos, entretanto estes estudos se restringem ao diagnóstico de parasitas humanos. Os poucos dados disponíveis sobre a utilização de kits comerciais no diagnóstico de parasitoses de cães tem revelado resultados comparáveis aos obtidos no diagnóstico humano (DrYDEN et al., 2005).

Na Austrália, IRWIN (2002) relatou que o envolvimento dos médicos veterinários com as doenças parasitárias nas clínicas veterinárias de pequenos animais se restringe à prescrição de drogas antiparasitárias de amplo espectro, refletindo com isso a convicção equivocada desses clínicos de que o controle das infecções parasitárias em animais de companhia possa se restringir ao uso de drogas. $\mathrm{O}$ uso desnecessário de parasiticidas além de trazer conseqüências indesejáveis para a saúde dos animais é considerado a principal causa responsável pelo desenvolvimento de resistência.

Ao que tudo indica, no Brasil, o desinteresse em solicitar e realizar exames coprológicos e a prescrição indiscriminada de parasiticidas é também a regra. 
Entretanto, além das preocupações já apontadas é necessário ter ciência de que os custos dessa atitude são muito mais elevados para a sociedade brasileira. O pior deles, talvez seja a falsa sensação de segurança que faz com que muitos médicos veterinários se desobriguem de conhecer o ciclo evolutivo dos parasitas, sua epidemiologia e profilaxia e, sobretudo, que se abstenham do papel de agentes promotores de saúde pública responsáveis pela educação sanitária dos proprietários de animais.

\section{REFERÊNCIAS}

Amato-Neto, V.; Campos, R.; Pinto, P.L.S.L.; Matsubara, L.; BraZ, L.M.A.; MiYAMOTO, A.; Foster, R.; NAscimento, S.A.B.; SouzA, H.B.W.T.; MoreIrA, A.A.B. Avaliação da utilidade do coprotest para exame parasitológico de fezes. Revista do Hospital das Clínicas da Faculdade de Medicina da Universidade de São Paulo, v.44, n.4, p.153-155, 1989.

Ambroise-Thomas, P. Emerging parasite zoonoses: the role of host-parasite relationship. International Journal for Parasitology, v.30, n.12/13, p.1361-1367, 2000.

Araújo, F.R.; Crocci, A.J.; Rodrigues, R.G.C.; Avalhaes, J.S.; Miyoshi, M.I.;SAlgado, F.P.;SIlva, M.A.; Pereira, M.L. Contaminação de praças públicas de Campo Grande, Mato Grosso do Sul, Brasil, por ovos de Toxocara e Ancylostoma em fezes de cães. Revista da Sociedade Brasileira de Medicina Tropical, v.32, n.5, p.581-583, 1999.

AraúJo, F.R.; AraúJo, C.P.; WerneCK, M.R.; GÓRSKI, A. Larva migrans cutânea em crianças de uma escola em área do Centro-Oeste do Brasil. Revista de Saúde Pública, v.34, n.1, p. 84-85, 2000.

AraúJo, A.J.U.S.; KanAmura, H.Y.; Dias, L.C.S.; Gomes, J.F.; ARAÚJo, S.M. Coprotest ${ }^{\circledR}$ quantitativo: quantificação de ovos de helmintos em amostras fecais utilizando-se sistema de diagnóstico comercial. Jornal Brasileiro de Patologia e Medicina Laboratorial, v.39, n.2, p.115-124, 2003.

Asano, K.; Suzuki, K.; Matsumoto, T.; SAKai, T.; Asano, R. Prevalence of dogs with intestinal parasites in Tochigi, Japan in 1979, 1991 and 2002. Veterinary Parasitology, v.120, p.243-248, 2004.

AsH, L.R.; Oritel, T.C. Parasites: a Guide to Laboratory Procedures and Identification. Chigago: ASCP Press, 1987. 328p.

BLAGBURN, B.L.; LINDSAY, D.S.; VAUGHAN, J.L.; RIPPEY, N.S.; WRIGHT, J.C.; LYNN, R.C.; KELEH, W.J.; RITCHIE, G.C.; HEPLER, D.I. Prevalence of canine parasites based on fecal flotation. The Compendium on Continuing Education for the Practicing Veterinarian, v.18, n.5, p.483-509, 1996.

Bowman, D.D.; Hendrix, C.M.; LindSAY, D.S.; BARR, S.C. Feline Clinical Parasitology. Iowa: Iowa State University Press, 2002. 469p.

Bowman, D.D.; LynN, R.C.; Eberhard, M.L. Georgi's Parasitology for Veterinarians. 8.ed. St. Louis: Saunders, 2003. 422p

BugG, R.J.; Robertson, I.D.; ElLIOT, A.D.; THOMPSON, R.S. Gastrointestinal parasites of urban dogs in Perth,
Western Austrália. The Veterinary Journal. v.157, p.295-301, 1999.

Cabrera, M.; Canova, S.; Rosenzvit, M., Guarnera, E. Identification of Echinococcus granulosus eggs. Diagnostic Microbiology and Infectious Disease, v.44, n.1, p.29-34, 2002.

CAmpos-Júnior, D.; Elefant, G.R.; Silva, E.O.M.; Gandolf, L.; JACOB, C.M.A.; TofetI, A.; Pratesi, R. Freqüência de soropositividade para antígenos de Toxocara canis em crianças de classes sociais diferentes. Revista da Sociedade Brasileira de Medicina Tropical, v.36, n.4, 2003.

Cerqueira, F.L. Coprotest: metodologia confiável para exame parasitológico de fezes. LAES/HAES, v.9, n.51, p.5-12, 1988.

CHAN, M-S. The global burden of intestinal nematode infections - fifty years on. Parasitology Today, v.13, n.11, p.438-443,1997.

Cordero del CAmpIllo, M.; Rojo, V.F.A. Parasitologia Veterinária. Madrid:McGraw-Hill Interamericana,1999.968p.

CôRTes, V.A.; PAim, G.V.; Filho, R.A.A. Infestação por ancilostomídeos e toxocarídeos em cães e gatos apreendidos em vias públicas, São Paulo (Brasil). Revista de Saúde Pública, v.22, n.4, p.341-343, 1988.

COSTA, H.M.A.; GUIMARÃES, M.P.; LEITE, A.C.; LIMA, W.S. Distribuição de helmintos parasitos de animais domésticos no Brasil. Arquivo Brasileiro de Medicina Veterinária e Zootecnia, v.38, n.4, p.465-579, 1986.

Costa-Cruz, J.M.; Nunes, R.S.; Buso, A.G. Presença de ovos de Toxocara spp. em praças públicas da cidade de Uberlândia, Minas Gerais, Brasil. Revista do Instituto de Medicina Tropical de São Paulo, v.36, n.1, p.39-42, 1994.

DAVID, E.D.; LINDQUIST, W.D. Determination of the specific gravity of certain helminth eggs using sucrose density gradient centrifugation. Journal of Parasitology, n.68, p.916-919, 1982.

Dryden, M.W.;PAyne, P.A.;RIdLEY, R.;Smith, V. Comparison of common fecal flotation techniques for the recovery of parasite eggs and oocysts. Veterinary Therapeutics, v.6, n.1, p.15-28, 2005.

Dubey, J.P.; SPeer, C.A.; FAyer, R. Sarcocystosis of animals and man. Boca Raton: CRC Press, 2000. 215p.

ECKERT, J.; DePlAZES, P. Biological, epidemiological, and clinical aspects of echinococcosis, a zoonosis of increasing concern, Clinical Microbiology Reviews, v.17, n.1, p.107-135, 2004.

Eguía-Aguilar, P.; Cruz-Reyes, A.; Martínez-Maya, J.J. Ecological analysis and description of the intestinal helminthes present in dogs in Mexico City. Veterinary Parasitology, v.127, p.139-146, 2005.

EsteVeZ, E.G.; LEVINE, J.A. Examination of preserved stool specimens for parasites: lack of value of the direct wet mount. Journal of Clinical Microbiology, v.22, p.666-667, 1985.

Farias, L.N; Malgor, R.; Cassaravilla, C.; Bragança, C.; DE LA RUE, M. L. Echinococcosis in southern Brazil: efforts toward implementation of a control program in Santana do Livramento, Rio Grande do Sul. Revista do Instituto de Medicina Tropical de São Paulo, v.46, n.3, p.153-156, 2004.

FAYER, R. Presidential address. Global change and emerging infectious diseases. Journal of Parasitology, v.86, n.6, p.1174-1181, 2000 . 
FOREYT, W.J. Diagnostic parasitology. Veterinary Clinics of North America Small Animal Practice, v.19, p.979-1000, 1989.

Fontanarrosa, M.F.; VezZAni, D.; Basabe, J.; Eiras, D.F. An epidemiological study of gastrointestinal parasites of dogs from Southern Greater Buenos Aires (Argentina): age, gender, breed, mixed infections, and seasonal and spatial patterns. Veterinary Parasitology, v.136, p.283-295, 2005.

GARCIA, L.S. Pros and Cons of using preservatives for $\mathrm{O}$ $\&$ P fecal specimens. Clinical Microbiology Newsletter, v.17, p.164-167, 1995.

Garcia, L.S. Diagnostic Medical Parasitology. 4 ed. Washington: ASM Press, 2001. 1092p.

GeFFrAy, L. Infections associated with pets. La Revue de Médecine Interne, v.20, p.888-901, 1999.

Gennari, S.M.; Kasai, N.; PenA, H.F.J.; CORTEZ, A. Ocorrência de protozoários e helmintos em amostras de fezes de cães e gatos da cidade de São Paulo. Brazilian Journal of Veterinary Research and Animal Science, v.36, n.2, p.87-91, 1999.

GeORGI, J.R. Tapeworms. Veterinary Clinics of North America Small Animal Practice, v. 17, n.6, p.1285-1306, 1987.

Georgi, J.R.; Georgi, M.E. Canine Clinical Parasitology. St. Louis: Lea \& Febiger, 1992. 227p.

Gomes, J.F.; Hoshino-Shimizu, S.; Dias, L.C.; Araujo, A.J.; Castilho, V.L.; Neves, F.A. Evaluation of a novel kit (TF-test) for the diagnosis of intestinal parasitic infections. Journal of Clinical Laboratory Analisys, v.18, p.132-138, 2004.

Guimarães, A.M.; Alves, E.G.L.; Rezende, G.F.; Rodrigues, M.C. Ovos de Toxocara sp. e larvas de Ancylostoma sp. em praça pública de Lavras, MG.. Revista de Saúde Pública, v.39, n.2, p.293-295, 2005.

Habluetzel, A.; Traldi, G.; Ruggieri, S.; Attili, A.R.; Scuppa, P.; Marchetti, R.; Menghini, G.; Esposito, F. An estimation of Toxocara canis prevalence in dogs, environmental egg contamination and risk of human infection in the Marche region of Italy. Veterinary Parasitology, v.113, p.243-252, 2003.

Hoffmman, A.N. Prevalência de Echinococcus granulosus (Batsch, 1786) em cães urbanos errantes do município de Dom Pedrito (RS), Brasil. Ciência Rural, v.31, n.5, p.843-847, 2001.

InG, M.B.; Schantz, P.M.; TuRner, J.A. Human coenurosis in North America: case reports and review. Clinical Infectious Diseases, v.27, n.3, p.519-523, 1998.

IRWIN, P.J. Companion animal parasitology: a clinical perspective. International Journal for Parasitology, v.32, p.581-593, 2002.

Jordan, H.E.; Mullins, S.T.; StebBins, M.E. Endoparasitism in dogs: 21.583 cases (1981-1990). Journal of the American Veterinary Medical Association, v.203, n.4, p.547-549, 1993.

KALKOFEN, U.P. Hookworms of dogs and cats. Veterinary Clinics of North America Small Animal Practice, v.17, n.6, p.1341-1354, 1987.

LandmanN, J.K.; Prociv, P. Experimental human infection with the dog hookworm, Ancylostoma caninum. The Medical Journal of Australia, v.178, p.69-71, 2003.

McCARTHY, J.; MoORE, T.A. Emerging helminth zoonoses. International Journal for Parasitology, v.30, p.1351-1360, 2000.
McGlade, T.R.; Robertson, I.D.; Elliot, A.D.; Read, C.; THOMPSON, R.C. Gastrointestinal parasites of domestic cats in Perth, Western Australia. Veterinary Parasitology, v.117, n.4, p.251-262, 2003.

MacPherson, C.N.L. Human behavior and the epidemiology of parasitic zoonoses. International Journal for Parasitology, v.35, p.319-1331, 2005.

Milsten, T.C.; Goldsmid, J.M. The presence of Giardia and other zoonotic parasites of urban dogs in Hobart, Tasmania. Australian Veterinary Journal, v.72, p.154155, 1995.

Molina, C.P.; Ogburn, J.; Adegboyega, P. Infection by Dipylidium caninum in an infant. Archives of Pathology $\mathcal{E}$ Laboratory Medicine, v.127, p.157-159, 2003.

Monis, P.T.; Andrews, R.H.; MAYrhOFER, G.; Ey, P.L. Genetic diversity within the morphological species Giardia intestinalis and its relationship to host origin. Infection, Genetics and Evolution, v.3, p.29-38, 2003.

Monis, P.T.; Thompson, R.C.A. Cryptosporidium and Giardia-zoonoses: fact or fiction? Infection, Genetics and Evolution, v.3, p.233-344, 2003.

Moore, D.P. Neosporosis in South America. Veterinary Parasitology, v.127, p.87-97, 2005.

Moraes, M.A.P.; Sobreira, M.N.M.; Medeiros Filho, P.; TAVARES, A.C.; Gomes, M.I. Hidatidose policística: cisto hidático calcificado, simulando neoplasia mesentérica, descoberto acidentalmente. Revista da Sociedade Brasileira de Medicina Tropical, v.4, n.36, p.519-521, 2003.

Oliveira-Sequeira, T.C.G.; Amarante, A.F.; Ferrari, T.B.; NunEs, L.C. Prevalence of intestinal parasites in dogs from São Paulo State, Brazil. Veterinary Parasitology, v.103, p.19-27, 2002.

Overganuw, P.A.M. Aspects of Toxocara epidemiology: toxocarosis in dogs and cats. Critical Reviews in Microbiology, v.23, p.233-251, 1997.

PARsons, J.C. Ascarid infections of cats and dogs. Veterinary Clinics of North America Small Animal Practice, v.17, n.6, p.1307-1340, 1987.

Perry, J.L.; Mathews, J.S.; Miller, G.R. Parasite detection efficiencies of five stool concentration systems. Journal of Clinical Microbiology, v.28, n.6, p.1094-1097, 1990.

Ponce-Macotela, M.; Peralta-Abarca, G.E.; Martinez-GoRDILlo, M.N. Giardia intestinalis and other zoonotic parasites: Prevalence in adult dogs from the southern part of Mexico City. Veterinary Parasitology, v.131, n.1-2, p.1-4, 2005.

Plant, M.; Zimmerman, E.M.; Goldstein, R.A. Health hazards to humans associated with domestic pets. Annual Review of Public Health, v.17, p.221-245, 1996.

Rabello, A.L.; ROCHA, R.S.; OLIVEIRA, J.P.M.; KATZ, N.; LAMBERTUCCI, J.R. Stool examination and rectal biopsy in the diagnosis and evaluation of therapy of schistosomiasis mansoni. Revista do Instituto de Medicina Tropical de São Paulo, v.34, n.6, p.601-608, 1992.

RAether, W.; HÄNel, H. Epidemiology, clinical manifestations and diagnosis of zoonotic cestode infections: an update. Parasitology Research, v.91, p.412-438, 2003.

Ramírez-Barrios, R.A.; Barboza-Mena, G.; Munoz, J.; Angulo-Cubillan, F.; Hernandez, E.; Gonzalez, F.; Escalona, F. Prevalence of intestinal parasites in dogs under veterinary care in Maracaibo, Venezuela. Veterinary Parasitology, v.121, p.11-20, 2004. 
ReY, L. Bases da parasitologia médica. Rio de Janeiro: Guanabara Koogan, 1992. 349p.

RoberTsOn, I.D.; IRWIN, P.J.; LyMBeRY, A.J.; THOMPSON, R.C. The role companion animals in the emergence of parasitic zoonoses. International Journal for Parasitology, v.30, p.1369-1377, 2000.

ROBERTSON, I.D.; THOMPSON, R.C. Enteric parasitic zoonoses of domesticated dogs and cats. Microbes and Infection, v.4, p.867-873, 2002.

Santarém, V.A.; Sartor, I.F.; Bergamo, F.M.M. Contaminação, por ovos de Toxocara spp., de parques de praças públicas de Botucatu, São Paulo, Brasil. Revista da Sociedade Brasileira de Medicina Tropical, v.31, n.6, p.529-532, 1998.

Scaini, C.J.; Toledo, R.; Lovatel, R.; Dionello, M A.; Gatti, F A.; Susin, L.; SignORINI, V.R.M. Contaminação ambiental por ovos e larvas de helmintos em fezes decães na área central de Balneário Cassino, Rio Grande do Sul. Revista da Sociedade Brasileira de Medicina Tropical, v.36, n.5, p. 617-619, 2003.

SHIN, J.W.; LIAO, W.T. Humoral immune response to Dipylidium caninum infection of stray dogs in Taiwan. Veterinary Parasitology, v.104, p.351-356, 2002.

Moreira-Silva, S.F.; LeÃo, M.E.; MendonçA, H.F.S. Prevalence of anti-Toxocara antibodies in a random sample of inpatients at a children's hospital in Vitória, Espírito Santo, Brazil., Revista do Instituto de Medicina Tropical de São Paulo, v.40, n.4, p.263-264, 1998.

Thompson, R.C.A. Veterinary parasitology: looking to the next millennium. Parasitology Today, v.15, n.8, p.320-325, 1999.
THOMPSON, R.C.A. The zoonotic significance and molecular epidemiology of Giardia and giardiasis. Veterinary Parasitology, v.126, n.1/2, p.15-35, 2004.

ThOMPSON, R.C.A.; LymberY, A.J.; HobBS, R.P. Teaching of parasitology to students of veterinary medicine and biomedical sciences. Veterinary Parasitology, v.108, n.4, p.283-290, 2002.

Truant, A.L.; Elliot, S.H.; Kelly, M.T.; Smith, J.H. Comparison of formalin-ethyl ether sedimentation, formalin-ethyl acetate sedimentation, and zinc sulfate flotation techniques for detection of intestinal parasites. Journal of Clinical Microbiology, v.13, n.5, p.882-884, 1981.

Urquhart, G.M.; Armour, J.; Duncan, J.L.; Dunn, A.M.; Jennings, F.W. Parasitologia Veterinária. 2.ed. Rio de Janeiro: Guanabara Koogan, 1996. 273p.

Velho, P.E.N.F.; FARIA, A.V.; CinTRA, M.L. Larva Migrans: a case report and review. Revista da Sociedade Brasileira de Medicina Tropical, v.45, n.3, p.167-171, 2003.

WHO. The world health report 1996. Fighting Disease Fostering Development. Geneva: World Health Organization, 1996.

Wilson, R.B. Cryptosporidiosis in a pup. Journal of the American Veterinary Medical Association, v.183, n 9, p.1005-1006, 1983.

XIAO, L.; RyAN, U.M. Cryptosporidiosis: an update in molecular epidemiology. Current Opinion in Infectious Diseases, v.17, n.5, p.483-490, 2004.

Recebido em 10/4/06

Aceito em 15/3/07 